reduced to zero and response rates to $T+\bar{L}$ and $\bar{T}+L$ systematically decline. Thus, there are differences in both the reinforcement and response rate parameters of the two test procedures. Either of these could account for the present findings. In Hamm and Meltzer's (1973) experiment with pigeons, results indicated a clear demonstration of negative summation but not positive summation. The authors hypothesized that the magnitude of difference in response rates between the two $S D_{S}$ and the $S \Delta$ were not sufficient to produce positive summation. In view of the results of the present experiment, this seems unlikely since the difference in response rates controlled by the two $S^{D_{S}}$ and $S \Delta$ were quite large for both S-5 and S-6.

One possible explanation for the failure to obtain response or positive summation in baseline testing situations is related to the particular reinforcement schedule associated with each component of the multiple schedule. Thus, if the pigeons in Hamm and Meltzer's (1973) experiment and in the present experiment were learning to associate a particular $\mathrm{S}^{\mathbf{D}}$ with a reinforcement density of about 60 reinforcers per hour, then a compound of the $S^{D_{S}}$ would signal a density of approximately 120 reinforcers per hour, if incentive values are additive. But, according to the data presented by Catania and Reynolds (1968), pigeons on VI schedules do not show much increase in response rate above a reinforcement density of approximately 60 reinforcers per hour. Thus, a significant increase in response rate would not be predicted for a compound in which each stimulus was associated with a reinforcement density of 60 reinforcers per hour unless the extinction test procedure was used. Preliminary tests now being conducted indicate that positive summation can be obtained in baseline testing with pigeons on a VI 3-min schedule. While to these authors' knowledge no data has been compiled for rats on VI schedules similar to that of Catania and Reynolds, it has been observed in this lab that rats will increase response rate when reinforcement density is increased above 60 reinforcers per hour. This could account for the differences between data reported for rats and the results of the present experiment with pigeons.

\section{REFERENCES}

Catania, A. C., \& Reynolds, G. S. A quantitative analysis of the responding maintained by interval schedules of reinforcement. Journal of the Experimental A nalysis of Behavior, 1968, 11. 327-383.

Hamm, R. J., \& Meltzer, D. A test for both positive and negative response summation in the pigeon. Bulletin of the Psychonomic Society, 1972, 2, 433-435.

Meltzer, D., \& Freeman, B. J. Maintenance of response summation under conditions of minimum stimulus intensity. Psychonomic Science, 1971, 22, 287-289.

Miller, L. Compounding of discriminative stimuli from the same and different modalities. Journal of the Experimental A nalysis of Behavior, 1971, 16, 337-342.

Miller, L., \& Ackley, R. Summation of responding maintained by fixed-inverval schedules. Journal of the Experimental A naly sis of Behavior, 1970, 13, 199-203.

Weiss, S. J. Summation of response strengths instrumentally conditioned to stimuli in different modalities. Journal of Experimental Psychology, 1964, 68, 151-155.

Weiss, S. J. Attentional processes along a composite stimulus continuum during free-operant summation. Journal of Experimental Psychology, 1969, 82, 22-27

Wolf, M. M. Some effects of combined S'́s. Journal of the Experimental Analysis of Behavior, 1970, 13, 199-203.

(Received for publication May 22, 1974.)

Bulletin of the Psychonomic Society

1974, Vol. 4 (2A), 97-99

\title{
Reinforcement schedule preference of a raccoon (Procyon lotor)*
}

\author{
GLEN D. KING, ROBERT W. SCHAEFFER $\dagger$ \\ Auburn University, Auburn, Alabama 36830 \\ and \\ STEPHEN C. PIERSON \\ University of Minnesota, Minneapolis, Minnesota 55455
}

\begin{abstract}
A raccoon, given a choice to respond for food pellets on either a FI 30-sec schedule or a FR schedule, the response requirement of which was systematically varied, preferred the FR schedule only when the rate of reinforcement on the FR schedule exceeded the rate of reinforcement on the FI schedule by a factor of two. The highly efficient responding of the raccoon on the FI schedule was noted as encouragement for using this species more frequently in operant learning paradigms.
\end{abstract}

A cornucopia of data has been collected concerning the performance of standard laboratory Ss under the contingencies of various reinforcement schedules. However, very little research time has been allocated to investigate the simple preference for reinforcement schedules or the parameters which determine simple schedule preference.

* Supported in part by Public Health Research Grants MG-08775 and MH-12025 from the National Institute of Mental Health, R. W. Schaeffer, principal investigator.

tRequests for reprints should be sent to Glen D. King, Department of Psychology, Auburn University. Appreciation is extended to Robert Bobbitt for competent and reliable assistance in the collection of this data.
The utilization of data from prosaic laboratory Ss such as the albino rat and the college sophomore in psychology has often been criticized. The raccoon, although not new to psychology as an experimental S (e.g., Johnson, 1961; Johnson \& Michels, 1958, 1961; and Hitchcock, Michels, \& Brown, 1963 employed this animal in double alternation and discrimination learning tasks), can readily be employed as a S, provided only that slight modifications are made of existing laboratory manipulanda which must be strengthened against the vigorous barrage of responding of which the raccoon is capable. The present study was undertaken simply to 
Table 1

Percent of Reinforcements Earned on Each Schedule During the Last 4 Days of Each Experimental Phase

\begin{tabular}{|c|c|c|c|c|}
\hline \multirow[b]{2}{*}{ Days } & \multicolumn{2}{|c|}{$\begin{array}{l}\text { Phase } 1 \\
\text { Training }\end{array}$} & \multicolumn{2}{|c|}{$\begin{array}{l}\text { Phase } 2 \\
\text { Choice }\end{array}$} \\
\hline & FR 60 & FI 30 & FR 60 & FI 30 \\
\hline 1 & 50 & 50 & 0 & 100 \\
\hline 2 & 50 & 50 & 0 & 100 \\
\hline 3 & 50 & 50 & 0 & 100 \\
\hline \multirow[t]{2}{*}{4} & 50 & 50 & 18 & 82 \\
\hline & \multicolumn{2}{|c|}{$\begin{array}{l}\text { Phase } 3 \\
\text { Training }\end{array}$} & \multicolumn{2}{|c|}{$\begin{array}{c}\text { Phase } 4 \\
\text { Choice }\end{array}$} \\
\hline Days & FR 20 & FI 30 & FR 20 & FI 30 \\
\hline 1 & 50 & 50 & 0 & 100 \\
\hline 2 & 50 & 50 & 1 & 99 \\
\hline 3 & 50 & 50 & 0 & 100 \\
\hline 4 & 50 & 50 & 0 & 100 \\
\hline
\end{tabular}

provide data on the preferential behavior of the raccoon as regards schedules of reinforcement. More specifically, the purpose of the experiment was to determine the raccoon's pepreference for response-dependent as opposed to time-dependent schedules of reinforcement.

\section{METHOD}

\section{Subjects}

The $S$ was a $18-1 b$ male raccoon, approximately 12 months old, that had been obtained at 4 months of age from a central Florida wildlife dealer. The raccoon was housed in a Wahmann Model LC-110B dog cage, which was equipped with a water reservoire supplied by a constantly running water source. S was maintained under conditions of constant illumination, termperature, and humidity. During both experiments, $\mathrm{S}$ was fed only once a day, immediately following each experimental session. The daily meal consisted of approximately $240 \mathrm{~g}$ of Purina Cat Chow and two apples. This food regimen ensured that $S$ would maintain food-reinforcement instrumental responding throughout the experimental sessions.

\section{Apparatus \\ A primate response-stimulus panel from a Foringer Model 1104 operant conditioning chamber was bolted onto the front of the home cage at the beginning of each session and removed at the termination of each session. This panel had two levers, a stimulus light directly above each lever, and a food magazine. Apertures cut in the wire mesh cage front allowed both levers and the food magazine to project into the cage space when the response-stimulus panel was mounted flush with the cage front. During each test session, $\mathrm{S}$ was separated from the water supply by inserting into the cage a metal panel which partitioned the living space into two equal halves. Illumination of cue lights, delivery of food-pellet reinforcements, and recording of responses were programmed by standard relay circuitry. White noise was used to mask any discriminative sounds produced by the programming equipment.}

\section{Procedure}

Two experiments were performed. In all phases of both experiments, as $S$ faced the response-stimulus panel, the FI 30-sec schedule was programmed on the lever to the S's right and the FR schedules were programmed on the lever to the S's left. The cue light above each lever was used as an $S^{D}$ to signal reinforcement availability on each lever.

Experiment 1. This experiment included four phases: a training phase, choice phase, retraining phase, and a second choice phase. After $\mathrm{S}$ was shaped to respond on a CRF schedule on both levers for 200-mg standard formula Noyes monkey pellets, the training phase was begun. In this phase, $S$ responded to a FI 30 -sec schedule on the right bar, and to a FR 30 schedule on the left bar. These schedule conditions remained in effect for 29 days, at which time it was determined that $S$ emitted approximately 60 responses per $30 \mathrm{sec}$ on the FR schedule. Consequently, the FR 30 schedule was increased to FR 60, while holding the FI 30-sec schedule constant, so as to equalize the rate of reinforcement on both the FI 30-sec and FR 60 schedules. This condition was continued for 8 days, subsequent to which the choice phase began and continued for 4 days.

During the training phase, only one reinforcement schedule was operative at any given time, and $\mathrm{S}$ was required to earn 50 consecu tive reinforcements on one schedule, then 50 consecutive reinforcements on the other schedule, after which the session was terminated. The schedule initially presented to $S$ in each test session was alternated daily.

In Phase 2, both schedules of reinforcement were concurrently operative and freely available for $S$ to choose between them. However, the first response on either lever rendered the other lever inoperative for a period of $2 \mathrm{~min}$. During this 2-min period, reinforcements could be obtained only on the lever initially chosen. At the end of each 2-min period, both levers became inoperative and the cue lights were extinguished for a period of $10 \mathrm{sec}$, after which another choice period between both levers became available. This sequence of events was continued throughout all choice periods in both experiments.

Phase 3 consisted of a return to training for 9 days, with the FR requirement set at 20 responses per reinforcement and the FI schedule held constant at $30 \mathrm{sec}$. The choice procedure in Phase 3 was the same as the choice procedure in Phase 2 and was continued for 4 days.

Experiment 2. Experiment 2 was designed to investigate S's relative preference for the two reinforcement schedules as the FR requirement was varied and the FI schedule was held constant at $30 \mathrm{sec}$. In each daily session, $\mathrm{S}$ was permitted to earn 100 reinforcements. In each session, $S$ was initially presented with either the FI schedule or the FR schedule, with the sequence alternating daily, and permitted to earn 20 consecu tive reinforcements on each schedule. Following this, $\mathrm{S}$ was permitted to choose between the concurrently and freely available schedules for the remaining 60 reinforcements in the session. As in the choice phase in Experiment 1, a choice on one schedule prevented responding on the other for a period of $2 \mathrm{~min}$, followed by a 10 -sec period in which neither schedule was available. The FI schedule was held constant at FI $30 \mathrm{sec}$ throughout this experiment, but the FR schedule was varied as follows: FR 20 for 4 days; FR 30 for 4 days; FR 40 for 4 days; FR 30 for 2 days; FR 40 for 4 days; FR 50 for 6 days; FR 20 for 5 days; FR 30 for 6 days; and FR 40 for 4 days.

\section{RESULTS AND DISCUSSION}

\section{Experiment 1}

During the first training session, the rate of reinforcement (pellets earned per minute) averaged 1.45 for the FR 60 schedule and 1.90 for the FI 30-sec schedule. Following the choice phase, when the FR requirement was reduced to FR 20 in the second training session, rates of reinforcement of 2.08 for the FR and 1.83 for the FI schedules were obtained. The percent of reinforcements earned for the last 4 days on 
Table 2

Mean Rate of Reinforcement (Pellets Earned Per Minute) for Each Reinforcement Schedule Across the Last 4 Days in the Initial Training Period of Each Experimental Phase

\begin{tabular}{cccccccccc}
\hline & \multicolumn{10}{c}{ Phases } \\
\cline { 2 - 11 } Schedule & FR 20 & FR 30 & FR 40 & FR 30 & FR 40 & FR 50 & FR 20 & FR 30 & FR 40 \\
\hline FR & 5.00 & 3.72 & 3.50 & 2.08 & 2.18 & 1.85 & 4.00 & 3.70 & 2.25 \\
FI & 2.00 & 1.90 & 2.00 & 1.90 & 1.85 & 1.70 & 1.70 & 1.83 & 1.90 \\
\hline
\end{tabular}

Table 3

Percent of Reinforcements Earned on Each Schedule Over the Last 4 Days of Each Experimental Phase

\begin{tabular}{|c|c|c|c|c|c|c|c|c|c|c|c|c|c|c|c|c|c|c|}
\hline \multirow[b]{2}{*}{ Days } & \multicolumn{2}{|c|}{$\begin{array}{c}\text { Phase } 1 \\
\text { FR } 20 \\
\end{array}$} & \multicolumn{2}{|c|}{$\begin{array}{r}\text { Phase } 2 \\
\text { FR } 30\end{array}$} & \multicolumn{2}{|c|}{$\begin{array}{r}\text { Phase } 3 \\
\text { FR } 40\end{array}$} & \multicolumn{2}{|c|}{$\begin{array}{r}\text { Phase } 4 \\
\text { FR } 30\end{array}$} & \multicolumn{2}{|c|}{$\begin{array}{r}\text { Phase } 5 \\
\text { FR } 40\end{array}$} & \multicolumn{2}{|c|}{$\begin{array}{r}\text { Phase } 6 \\
\text { FR } 50\end{array}$} & \multicolumn{2}{|c|}{$\begin{array}{r}\text { Phase } 7 \\
\text { FR } 20\end{array}$} & \multicolumn{2}{|c|}{$\begin{array}{r}\text { Phase } 8 \\
\text { FR } 30\end{array}$} & \multicolumn{2}{|c|}{$\begin{array}{r}\text { Phase } 9 \\
\text { FR } 40\end{array}$} \\
\hline & FR & FI & FR & FI & FR & FI & FR & FI & FR & FI & FR & FI & FR & FI & FR & FI & FR & FI \\
\hline 1 & 100 & 0 & 100 & 0 & 69 & 31 & 50 & 50 & 27 & 73 & 9 & 91 & 54 & 46 & 89 & 11 & 23 & 77 \\
\hline 2 & 100 & 0 & 98 & 2 & 22 & 78 & 47 & 53 & 17 & 83 & 1 & 99 & 76 & 24 & 67 & 33 & 84 & 16 \\
\hline 3 & 100 & 0 & 100 & 0 & 27 & 73 & $*$ & $*$ & 0 & 100 & 3 & 97 & 82 & 18 & 34 & 66 & 40 & 60 \\
\hline 4 & 100 & 0 & 100 & 0 & 0 & 100 & $*$ & $*$ & 0 & 100 & 0 & 100 & 76 & 24 & 56 & 44 & 20 & 80 \\
\hline
\end{tabular}

${ }^{*} S$ was only run for 2 days in this condition.

each schedule for each phase of this experiment, presented in Table 1, indicates that $\mathrm{S}$ preferred to earn reinforcements on the FI $30-$ sec schedule not only when the reinforcement density was greater on the FI schedule, but even when the rate of reinforcement on the FR schedule was greater than for the FI schedule. Thus, S's clear preference for the FI schedule could not have been determined by rate of reinforcement alone.

\section{Experiment 2}

This study investigated the relationship between relative rates of reinforcement on the FR and FI schedules by varying the FR requirement while the FR schedule remained constant. The mean relative rates of reinforcement during the last 4 days of each phase are presented in Table 2 . These rates represent mean pellets earned per minute when $S$ was required in the initial part of each daily session to obtain 20 reinforcements on each schedule prior to being given a choice between the two schedules. In all phases, the rate of reinforcement was higher for the FR schedule than for the FI schedule, with FR rate of reinforcement inversely related to FR requirement. Comparisons of Tables 2 and 3 show that $S$ preferred the FR schedules only when the rate of reinforcement on the FR schedule was more than approximately twice as great as the FI schedule. The failure to replicate precisely the rate of reinforcement values and schedule preferences across phases (cf. Phases 1 and 7;2 and 4) renders this conclusion slightly less emphatic than desired; nonetheless, the conclusion is clearly warranted. It is unlikely that this failure to gain exact replication within the experiment was due to sequential effects. Rather, it appears that other parameters of reinforcement schedules apart from reinforcement rates may influence schedule preference.
More specifically, it may be that schedules of reinforcement have differential, inherent aversive properties associated with the response and/or time requirements of the schedule.

Behavioral confirmation of the inference that reinforcement schedules may have inherently aversive properties was obtained dramatically in the present experiment when the raccoon was forced to respond on the FR schedule during the training periods of each daily session. When presented with the FR schedule, the S became hyperactive, vocalized high pitched sounds, exhibited stereotyped pacing, and refused to respond on the FR lever for prolonged periods of time. By comparison, toward the end of the experiment, the $S$ consistently emitted less than five responses per reinforcement on the FI 30-sec schedule, and showed none of the hyperactive agitated behaviors described above for the FR schedule. In addition to suggesting that the FI schedule was much less aversive than the FR schedule to the S, S's behavior on the FI schedule indicated that raccoons (1) may be capable of highly efficient temporal discrimination on FI schedules, and (2) may be important experimental Ss for further investigations in operant learning paradigms.

\section{REFERENCES}

Hitchcock, L., Michels, K. M., \& Brown, D. R. Discrimination learning: Squirrels vs raccoons. Perceptual \& Motor Skills, $1973,16,405-414$.

Johnson, J. I. Double alternation by raccoons. Journal of Comparative \& Physiological Psychology, 1961, 54, 248-251. Johnson, J. I., \& Michels, K. M. Discrimination of small intervals and objects by raccoons. Animal Behavior, 1958, 6, 164-170. Johnson, J. I., \& Michels, K. M. Learning sets and object-size effects on visual discrimination learning by raccoons. Journal of Comparative \& Physiological Psychology, 1958, 51, 376-379.

(Received for publication April 12, 1974.) 